\title{
Original
}

\section{Regional Levels of Brain Monoamines and Their Metabolites in the Development Phase of Hypertension in SHR}

\author{
Yumi KamiJo, Ichirou Fukasawa, Yuji Kivchi, Miyuki Hashimoto, \\ Hiroshi Akita, Katsuji Oguchi and Hajime Yasuhara
}

\begin{abstract}
Age-related changes in the content of monoamines and their metabolites in four regions of the brain, cortex $(\mathrm{CX})$, midbrain and thalamus (MB), hypothalamus (HT), and medulla oblongata (MO), were quantitatively assessed in spontaneously hypertensive rats (SHR) and Wistar-Kyoto rats (WKY) to determine the contribution of brain amines to the pathogenesis of hypertension. Seven-week-old SHR had a significantly higher concentration of noradrenaline (NA) in the CX, MB, and HT than age-matched WKY. On the other hand, at 12-weeks, NA contents in all four regions of SHR was significantly lower than in WKY. There was no difference in dopamine (DA) content in these four regions between 7-week-old SHR and age-matched WKY. But in 12-week-old SHR, DA content in CX and MB were significantly lower than in WKY. Seven-week-old SHR had significantly higher 5-HT concentration than WKY in the CX, MB, MO and HT, but at 12 weeks, 5-HT content in all these four brain regions of SHR were significantly lower than in WKY. The contents of MHPG and 5HIAA, which are metabolites of NA and 5-HT, respectively, in all four regions were lower in SHR than in WKY at 12 weeks. The contents of DOPAC and HVA, metabolites of DA, in the brain of 12-week-old SHR, also showed the same tendency. These results suggest that NA, DA and 5-HT in the brain may simultaneously affect the onset and development of hypertension in SHR.
\end{abstract}

Key words: noradrenaline, dopamine, serotonin, hypertension, SHR

\section{Introduction}

The spontaneously hypertensive rats (SHR) established by Okamoto and Aoki ${ }^{1)}$ are presently considered to be among the most suitable models for studying human essential hypertension. Various pathophysiological and biochemical findings have been obtained for SHR, but the mechanism of their hypertension remains to be elucidated. There are several theories of blood pressure regulation, such as the mosaic theory in which one pathogenesis of hypertension would be metabolism of abnormal central monoamines. Moreover, since Yamori et al. reported low concentration of noradrenaline (NA) in the brain stem of SHR, it has been suspected that catecholamines (CA) may be important in the development of hypertension. ${ }^{2)}$

We have studied the hypotensive effect of clorgyline, a selective inhibitor of MAO-A in normotensive rats") and SHR. ${ }^{3)}$ Blood pressure decreased after intraperitoneal (i.p.) or

Department of Pharmacology, Showa University School of Medicine, 1-5-8 Hatanodai, Shinagawa-ku, Tokyo 142, Japan. 
intracerebroventricular (i.c.v.) injection of clorgyline. Based on data obtained in these studies, we concluded that the observed hypotension might be related to the accumulation of centrally acting NA or serotonin $(5-\mathrm{HT})$ in the brain as a result of MAO-A inhibition by clorgyline. The present investigation was conducted to study the role of catecholamine in the onset of hypertension in SHR. Regional distribution of NA, DA, 5-HT and their metabolites in the brain was examined in young ( 7 week old) and adult (12 week old) SHR.

\section{Methods}

Male SHR and WKY from 5 to 14 weeks were used. The blood pressure and heart rate of SHR and WKY were measured in conscious animals by tail plethysmography with Natume KN-201-I.

To measure of NA, DA, 5-HT, and their metabolites, 7- or 12-week-old rats were sacrified by decapitation and the brains were removed as quickly as possible, placed on ice and dissected into seven regions according to the method of Glowinski and Iversen. ${ }^{5)}$ Four of the seven brain regions, the cerebral cortex (CX), midbrain and thalamus (MB), medulla oblongata (MO) and hypothalamus (HT) were assayed for amines and their metabolites. Those four regions were chosen for the following reasons: The $\mathrm{CX}$ receives projections from the brain stem. The MO and MB include important nucleis for blood pressure regulation (the locus coeruleus is rich in NE cells and the nucleus raphe is rich in 5-HT cells). The HT sends fibers to the vasomotor center in brain stem.

Samples of each region were homogenized in $0.12 \mathrm{M}$ sodium acetate buffer, $\mathrm{pH}$ 5.0, and then centrifuged at $15,000 \times \mathrm{g}$ for $20 \mathrm{~min}$ at $4^{\circ} \mathrm{C}$. The supernatant was hydrolyzed enzymatically with sulfatase. ${ }^{6)}$ After repeated centrifugation, the supernatant was washed with chloroform and then analyzed for NA, 3-methoxy-4-hydroxyphenylglychol (MHPG), DA, 3,4,dihydroxyphenylacetic acid (DOPAC), 5-HT, 5-hydroxyindolacetic acid (5-HIAA) and homovanillic acid (HVA) by high performance liquid chromatography with an electrochemical detector (HPLC-ECD). ${ }^{i s)}$

\section{Results}

As shown in Fig. 1, the blood pressure of SHR increased remarkably in the first 9 weeks and more slowly thereafter until 14 weeks. The blood pressure of WKY increased only slightly in the first 7 weeks, and was constant thereafter. Based on these results, we selected 7-week-old and 12-week-old SHR as animals representing the development and establishment phases of hypertension, respectively.

The contents of NA and MHPG in the brain regions are summarized in Table 1. At 7 weeks, NA levels in the CX, MB and HT of the SHR were significantly higher than those in the WKY, and in the MO region of the SHR they tended to be higher than in the WKY. In contrast, at 12 weeks, NA levels in all brain regions of the SHR examined in this study were significantly lower than those in the WKY. On the other hand, the level of MHPG, a metabolite of NA, was comparable to that in age-matched WKY, in all brain regions of the SHR at 7 weeks. At 12 weeks, those levels were significantly lower in SHR than in WKY in all the brain regions except the MB. In SHR at 12 weeks of age, NA levels in all brain regions tended to be lower than those at 7 weeks of age. On the contrary, WKY at 12 weeks of age tended to have higher NA levels in all the brain regions than at 7 weeks.

As shown in Table 2, at 7 weeks DA levels in all four SHR brain regions were com- 


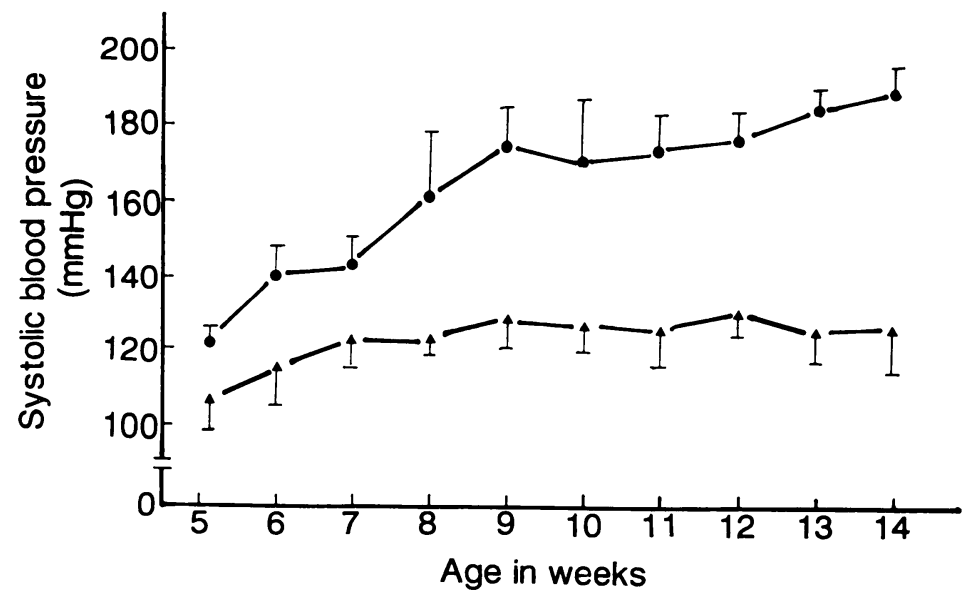

Fig. 1. Effect of age on systolic blood pressure in rats.

๑ : SHR, $\Delta$ : WKY, $(\mathrm{n}=5)$, Mean \pm S.D.

Table 1. The contents of NA and MHPG in SHR and WKY brain regions ( $\mathrm{ng} / \mathrm{g}$ tissue).

\begin{tabular}{|c|c|c|c|c|c|}
\hline & \multicolumn{2}{|r|}{ Midbrain + thalamus } & Medulla oblongata & Hypothalamus \\
\hline \multirow[t]{4}{*}{ NA } & SHR-7 & $631.0 \pm 130.3^{* *}$ & $722.2 \pm 119.1 * *$ & $956.2 \pm 672.9$ & $1798.1 \pm 238.8^{*}$ \\
\hline & WKY-7 & $334.2 \pm 79.3$ & $408.4 \pm 83.8$ & $369.8 \pm 151.2$ & $1303.4 \pm 331.6$ \\
\hline & SHR-12 & $393.7 \pm 83.4^{* *}$ & $513.6 \pm 140.2^{*}$ & $502.4 \pm 99.4^{*}$ & $1431.0 \pm 383.8^{*}$ \\
\hline & WKY-12 & $905.7 \pm 146.6$ & $1123.3 \pm 419.1$ & $752.9 \pm 170.1$ & $2312.6 \pm 850.8$ \\
\hline \multirow[t]{4}{*}{ MHPG } & SHR-7 & $184.8 \pm 30.1$ & $220.1 \pm 71.7$ & $274.8 \pm 43.1$ & $241.0 \pm 37.3$ \\
\hline & WKY-7 & $170.5 \pm 146.4$ & $258.3 \pm 204.3$ & $308.7 \pm 250.9$ & $352.9 \pm 279.1$ \\
\hline & SHR-12 & $117.2 \pm 15.8^{* *}$ & $147.0 \pm 26.1$ & $172.6 \pm 28.3^{*}$ & $141.4 \pm 30.2^{* *}$ \\
\hline & WKY -12 & $181.7 \pm 28.9$ & $228.5 \pm 58.3$ & $260.5 \pm 53.5$ & $269.6 \pm 68.9$ \\
\hline
\end{tabular}

WKY-7 ( $n=5)$, SHR-7, 12, WKY-7 $(n=6)$

Results are expressed as mean \pm S.D.

Significant differences from control values $* \mathbf{P}<0.05$. ** $\mathrm{P}<0.01$.

parable to those in age-matched WKY. However, at 12 weeks, DA levels in the CX and MB in the SHR were significantly lower than those in the WKY. The levels of DOPAC, a metabolite of DA, were comparable in all four SHR brain regions at 7 weeks of age to those in age-matched WKY. However, at 12 weeks of age, the levels of DOPAC in the CX, MB and MO were significantly lower in SHR than in WKY. The levels of HVA, another metabolite of DA, were comparable in all the four regions in the SHR to those in the WKY at 7 weeks. However, in the CX, the DA level in SHR was significantly lower at 12 weeks than that in WKY. In the SHR, DA levels in the CX and MB tended to be lower at 12 weeks than at 7 weeks. In contrast, DA levels in the CX, MB and HT in the WKY tended to be higher at 12 weeks than at 7 weeks.

The 5-HT and 5-HIAA contents in the brain are summarized in Table 3. In all brain regions examined, the contents of 5-HT in the SHR at 7 weeks of age were significantly higher than those in the WKY. However, at 12 weeks the 5-HT contents in all SHR brain 
Table 2. The contents of DA, DOPAC and HVA contents in SHR and WKY brain regions $(\mathrm{ng} / \mathrm{g}$ tissue).

\begin{tabular}{clcccc}
\hline & & Cortex & Midbrain +thalamus & Medulla oblongata & Hypothalamus \\
\hline DA & SHR-7 & $648.9 \pm 414.7$ & $176.0 \pm 164.5$ & $52.7 \pm 11.8$ & $1571.7 \pm 1047.6$ \\
& WKY-7 & $631.2 \pm 184.9$ & $69.7 \pm 32.3$ & $67.2 \pm 32.6$ & $1112.1 \pm 634.4$ \\
& SHR-12 & $415.1 \pm 88.8^{* *}$ & $94.2 \pm 28.3^{*}$ & $60.5 \pm 6.6$ & $1573.5 \pm 894.4$ \\
& WKY-12 & $957.1 \pm 258.3$ & $184.6 \pm 76.3$ & $70.8 \pm 10.9$ & $1877.5 \pm 1024.2$ \\
\hline \multirow{2}{*}{ DOPAC } & SHR-7 & $141.7 \pm 67.0$ & $169.4 \pm 86.4$ & $147.3 \pm 46.0$ & $620.1 \pm 283.5$ \\
& WKY-7 & $244.4 \pm 116.4$ & $175.1 \pm 106.4$ & $147.1 \pm 97.7$ & $463.7 \pm 185.1$ \\
& SHR-12 & $154.4 \pm 30.9 * *$ & $83.8 \pm 11.6^{*}$ & $60.6 \pm 7.2^{*}$ & $592.5 \pm 151.3$ \\
& WKY-12 & $270.8 \pm 50.7$ & $245.6 \pm 113.7$ & $219.1 \pm 148.9$ & $480.3 \pm 150.5$ \\
\hline HVA & SHR-7 & $133.8 \pm 28.2$ & $102.6 \pm 27.6$ & $92.0 \pm 23.5$ & $299.8 \pm 80.4$ \\
& WKY-7 & $153.6 \pm 40.2$ & $99.7 \pm 16.8$ & $132.0 \pm 42.6$ & $225.8 \pm 63.3$ \\
& SHR-12 & $85.9 \pm 18.9 *$ & $66.9 \pm 22.6$ & $58.0 \pm 14.4 *$ & $178.2 \pm 68.3$ \\
& WKY-12 & $115.6 \pm 20.5$ & $91.9 \pm 26.1$ & $90.3 \pm 28.9$ & $211.3 \pm 24.2$ \\
\hline
\end{tabular}

WKY-7 (n=5), SHR-7, 12, WKY-12 $(\mathrm{n}=6)$

Results are expressed as mean \pm S.D.

Significant differences from control values $* \mathrm{P}<0.05$. ${ }^{* *} \mathrm{P}<0.01$.

Table 3. The contents of 5-HT and 5-HIAA in SHR and WKY brain regions (ng/g tissue).

\begin{tabular}{clcccc}
\hline & & \multicolumn{1}{c}{ Cortex } & Midbrain +thalamus & Medulla oblongata & Hypothalamus \\
\hline 5-HT & SHR-7 & $779.2 \pm 226.2^{* *}$ & $839.1 \pm 172.7 * *$ & $804.0 \pm 234.2^{* *}$ & $885.6 \pm 183.9^{* *}$ \\
& WKY-7 & $180.5 \pm 46.8$ & $247.5 \pm 114.1$ & $293.4 \pm 127.0$ & $398.0 \pm 111.1$ \\
& SHR-12 & $233.3 \pm 19.7 * *$ & $409.3 \pm 157.2^{* *}$ & $294.1 \pm 85.6^{* *}$ & $470.5 \pm 63.6^{* *}$ \\
& WKY-12 & $1389.3 \pm 301.7$ & $1549.4 \pm 463.2$ & $1504.1 \pm 385.1$ & $1582.6 \pm 421.1$ \\
\hline \multirow{2}{*}{ 5-HIAA } & SHR-7 & $442.0 \pm 65.1^{* *}$ & $918.0 \pm 116.5 * *$ & $808.3 \pm 191.9$ & $771.5 \pm 146.4$ \\
& WKY-7 & $215.7 \pm 66.5$ & $562.5 \pm 65.8$ & $566.5 \pm 127.2$ & $602.5 \pm 81.8$ \\
& SHR-12 & $390.0 \pm 31.0^{*}$ & $758.0 \pm 128.9 * *$ & $676.8 \pm 213.7 * *$ & $735.4 \pm 205.9 * *$ \\
& WKY-12 & $897.0 \pm 168.0$ & $1426.5 \pm 401.6$ & $1367.5 \pm 381.7$ & $1630.8 \pm 486.8$ \\
\hline
\end{tabular}

WKY-7 $(n=5)$, SHR-7, 12, WKY-12 $(n=6)$

Results are expressed as mean \pm S.D.

Significant differences from control values $* \mathbf{P}<0.05$. ${ }^{* *} \mathbf{P}<0.01$.

regions were lower than those of the WKY. At 7 weeks, the 5-HIAA content, a metabolite of 5-HT, was significantly higher in the CX and MB of the SHR than in the WKY, whereas at 12 weeks, this was significantly lower in all brain regions examined. In the four brain regions, the contents of 5-HT tended to be lower in 12-week-old SHR than in 7week-old SHR, whereas the 5-HT contents tended to be higher in 12-week-old WKY than in 7-week-old WKY.

\section{Discussion}

The present study demonstrates the age-related changes in NA, DA, 5-HT and their metabolites in four brain regions of young and adult SHR, and compares them to the changes in WKY. 
In our results, NA levels in the brain of young and adult SHR were different from those of age-matched WKY, indicating the possibility that these changes in SHR might be related to the development of hypertension. This hypothesis is supported by the finding that 6hydroxydopamine (6-OH-DA), which destroys central adrenergic neurons, prevents the development of hypertension in SHR, renal and DOCA/ $\mathrm{NaCl}$ hypertensive rats. ${ }^{9,10)}$ Furthermore, in young SHR, serum DBH activity was higher than that in age-matched WKY.11.12) These studies indicate that increased NA in the brain plays an important role in the development of hypertension in SHR.

The posterior hypothalamus (PHT), which is thought to be a tonically active center of central blood pressure regulation, is important in the development of hypertension in SHR. The PHT projects fibers directly or indirectly to the vasomotor center located in the brain stem and these neurons modulate peripheral nerve activity. Electrical stimulation of the PHT of SHR increases arterial blood pressure and heart rate. ${ }^{13,14}$ Stimulation-induced release of ${ }^{3} \mathrm{H}-\mathrm{NA}$ was increased in the PHT of 10- to 12-week-old SHR. ${ }^{15)}$ Central administration of NA in the PHT produced significant pressor responses. ${ }^{16)}$ These results suggest the presence of pressor receptors in the PHT that respond to NA. However, the HT that we report here included the anterior hypothalamus (AHT) which is also thought to reduce the arterial blood pressure by controlling inhibition of central sympathetic tone. ${ }^{17)}$ Therefore no reliable information is available from the present study as to the detailed localization of a trigger in the HT. NA content in all brain regions was high in young SHR, but MHPG content in the brain of young SHR differed from than in WKY. NA contents in all brain regions of the adult SHR decreased with a concomitant decrease in MHPG content. These results suggest that the increase in the NA content of the respective brain regions of young SHR reflects both changes in NA turnover and changes in NA synthesis. It is possible to relate these general decreases in catecholamine levels to a progressive rise in blood presure. A previous study of NA turnover using $\alpha$-methyl-p-tyrosine showed no difference between young SHR and age-matched WKY in the PHT. ${ }^{18)}$ The increased contents might represent an increase in the granules in an active pool of NA. ${ }^{19)}$

Our results indicate similar brain DA content in young SHR and age-matched WKY. However, DA contents in the MB of young SHR tended to be higher than in age-matched WKY. Pretreatment with GBR-12909, a DA uptake-blocker, inhibits the development of hypertension. ${ }^{20}$ Other studies have shown different results. For example, administration of bromocriptine, a DA agonist, significantly decreases blood pressure. ${ }^{21)}$ No fall in blood pressure or heart rate was observed after intracerebroventricular injection of DA. ${ }^{22}$ Little is known about the role of the brain DA system in the regulation of blood pressure, but in our studies, there was a definite pattern in the age-related changes in DA and DA metabolite levels. The lower DA, DOPAC and HVA levels found in adult SHR compared to those in adult WKY suggested some change in DA levels occurring with age. Our results and those of others suggest that brain DA may be important in the development of hypertension.

In the present results, brain 5-HT content in young and adult SHR differed from those in age-matched WKY. Injection of 5-HT into the cerebral ventricles increased blood pressure, and these pressor responses were prevented by intraventricular injection of bromolysergic acid diethylamide and ketanserin, which are 5-HT receptor antagonists. ${ }^{23,24)}$ These studies suggest that brain 5-HT is also important in blood pressure regulation. In our studies, the content of 5-HT in the brain of young SHR was significantly higher than that 
in WKY, and helped to explain these hypotheses. Content of 5-HT in all brain regions of young SHR increased along with concomitant increase in 5-HIAA contents of CX and MB. Our studies indicated that the higher 5-HT content in the brain of young SHR was caused by both the change in 5-HT turnover and the change in 5-HT synthesis. The metabolism of 5-HT is selectively higher in the hypothalamus and brain stem of SHR. ${ }^{2 i)}$ It is difficult, however, to make a generalization regarding 5-HT metabolism in the brain regions examined in our study. We must conduct further anatomical, physiological and pharmacological studies to elucidate the mechanisms of the 5-HT system in blood pressure regulation.

In our studies, more generalized patterns of changes of NA, 5-HT and their metabolites with age were apparent in SHR and WKY. The contents of NA and 5-HT in the brain regions of young SHR were higher than those in young WKY and in adult SHR they were lower than in adult WKY. The content of MHPG, 5-HIAA, DOPAC and HVA in the brain of adult SHR were lower than in adult WKY. In conclusion, these results appear to support the hypothesis that brain NA, DA and 5-HT simultaneously influence the development of hypertension in SHR.

\section{References}

1) Okamoto $\mathrm{K}$ and Aoki K: Development of strain of spontaneously hypertensive rats. Jpn Circ J, 27: 282-293 (1983)

2) Yamori $\mathrm{Y}$, Lovenberg $\mathrm{W}$ and Joerdsma AS: Norepinephrine metabolism in brain stem of spontaneously hypertensive rats. Science, 170: 544-546 (1970)

3) Yamamoto $\mathbf{M}$, Hashimoto $\mathbf{M}$, Yamada $F$, Nonoyama $T$, Fukazawa I, Oguchi $\mathbf{K}$ and Yasuhara $\mathbf{H}$ : Hypotensive effects of the selective MAO-A inhibitors. J Showa Med Assoc, 48: 455-458 (1988)

4) Fukasawa I, Kiuchi Y, Yamada F, Hashimto M, Oguchi K and Yasuhara H: The central mechanism of the hypotensive effects of clorgyline and deprenyl in spontaneously hypertensive rats. Biogen Amines, 6: 549-557 (1989)

5) Glowinski J and Iversen LL: Regional studies of catecholamines in the rat brain-1. The disposition of ${ }^{3} \mathrm{H}$-norepinephrine, ${ }^{3} \mathrm{H}$-dopamine and ${ }^{3} \mathrm{H}$-dopa in various regions of the brain. $J$ Neurochem, 13: 655-669 (1966)

6) Warnhoff $M$ : Simultaneous determination of norepinephrine, dopamine, 5-hydroxytryptamine and their main metabolites in rat brain using high-performance liquid chromatography with electrochemical detection. J Chromatogr, 307: 271-281 (1984)

7) Kilts CD, Breese GR and Mailman RB: Simultaneous quantification of dopamine, 5-hydroxytryptamine and four metabolically related compounds by means of reversed-phase high-performance liquid chromatography with electrochemical detection. J Chromatogr, 225: 347-357 (1981)

8) Tani Y, Kataoka Y, Sakurai Y, Yamashita K, Ushino M and Ueki S: Changes of brain monoamine contents in three models of experimentally induced muricide in rats. Pharmacol Biochem Behavior, 26: $725-729$ (1987)

9) Finch $L$, Haeusler $G$ and Thoenen $H$ : Failure to induce experimental hypertension in rats after intraventricular injection of 6-hydroxydopamine. Br J Pharmacol, 44: 356-357 (1972)

10) Buuse M, Kloet ER, Versteeg DG and Jong W: Regional brain catecholamine levels and the development of hypertension in the spontaneously hypertensive rat: The effect of 6-hydroxydopamine. Brain Res, 301: 221-229 (1983)

11) Nagatsu T, Kato T, Numata (Sudo) Y, Ikuta K, Umezawa H, Matsuzaki $\mathbf{M}$ and Takeuchi $T$ : Serum dopamine $\beta$-hydroxylase activity in developing hypertensive rats. Nature, 251: 630-631 (1974)

12) Nakamura $K$ and Nakamura $K$ : Role of brain stem and spinal noradrenergic and adrenergic neurons in the development and maintenance of hypertension in spontaneously hypertensive rats. Arch Pharm, 305: 127-133 (1978)

13) Calaresu FR and Thomas MR: Electrophysiological connections in the brain stem involved in cardiovascular regulation. Brain Res, 87: 335-338 (1975)

14) Juskevich JC, Robinson DS and Whitehorn D: Effect of hypothalamic stimulation in spontaneously hypertensive and Wistar-Kyoto rats. Eur J Pharmacol, 51: 429-439 (1978) 
15) Meldrum MJ and Westfall TC: Comparison of norepinephrine release in hypertensive rats: 1 Hypothalamic and brain stem tissue. Clin Exp Hypertens $(A), 201-219$ (1986)

16) Zawoiski EJ: Central actions of norepinephrine, phentolamine and 6-hydroxydopamine in spontaneously hypertensive rats. Arch Int Pharmacodyn, 247: 103-118 (1980)

17) Benarroch EE, Balda MS, Finkielman S and Nahmod VE: Neurogenic hypertension after depletion of norepinephrine in anterior hypothalamus induced by 6-hydroxydopamine administration into the ventral pons: role of serotonin. Neuropharmacology, 22: 29-34 (1983)

18) Sherry R, Winternitz J, Wyss JW and Oparil S: The role of the posterior hypothalamic area in the pathogenesis of hypertension in the spontaneously hypertensive rat. Brain Res, 324: 51-58 (1984)

19) Oltmans GA, Olsauskas R and Comaty JE: Hypothalamic catecholamine systems in genetically obese mice (obob): decreased sensitivity to reserpine treatment. Neuropharmacology, 19: 25-33 (1979)

20) Buuse M, Versteeg DHG and Jong W: Brain catecholamines and the development of hypertension in spontaneously hypertensive rats. J Hypertens [Suppl], 3: 159-161 (1986)

21) Nagahama S, Chen Y-F and Oparil S: Mechanism of depressor effect of bromocryptine in the spontaneously hypertensive rat. J Pharmacol Exp Ther, 228: 370-375 (1984)

22) Haeusler $\mathbf{G}$, Gerold $\mathbf{M}$ and Thoenen $\mathbf{H}$ : Cardiovascular effect of 6-hydroxydopamine injected into a lateral brain ventricle of the rat. Naunyn Schmiedebergs Arch Pharmacol, 274: 211-228 (1972)

23) Lambert GA, Friedman E, Buchweitz E and Gershon S: Involvement of 5-hydroxytryptamine in the central control of respiration, blood pressure and heart rate in anaesthetized rat. Neuropharmacology, 17: 807-813 (1978)

24) Kushiro T, Kobayashi F, Satoh K, Kawai S, Hashida J, Kobayashi Y and Kajiwara N: The role of serotonergic nervous system in blood pressure regulation in spontaneously hypertensive rats. J Hypertens, [Suppl] 3: 221-222 (1986)

25) Koulu M, Saavedra JM, Niwa M, Scheinin M and Linnoila M: Association between increased serotonin metabolism in rat brain stem nuclei and development of spontaneous hypertension. Brain Res, 371: 177-181 (1986)

[Received September 11, 1989: Accepted October 20, 1989] 